ARTICLE

\title{
Prominent radiative contributions from multiply- excited states in laser-produced tin plasma for nanolithography
}

\author{
F. Torretti, ${ }^{1,2}$, J. Sheil ${ }^{1}$, R. Schupp (10 1, M. M. Basko ${ }^{3}$, M. Bayraktar (1) 4, R. A. Meijer ${ }^{1,2}$, S. Witte ${ }^{1,2}$, W. Ubachs (1) 1,2, \\ R. Hoekstra ${ }^{1,5}$, O. O. Versolato (i) ${ }^{1 凶}$, A. J. Neukirch ${ }^{6} \&$ J. Colgan (i) ${ }^{6 凶}$
}

Extreme ultraviolet (EUV) lithography is currently entering high-volume manufacturing to enable the continued miniaturization of semiconductor devices. The required EUV light, at $13.5 \mathrm{~nm}$ wavelength, is produced in a hot and dense laser-driven tin plasma. The atomic origins of this light are demonstrably poorly understood. Here we calculate detailed tin opacity spectra using the Los Alamos atomic physics suite ATOMIC and validate these calculations with experimental comparisons. Our key finding is that EUV light largely originates from transitions between multiply-excited states, and not from the singly-excited states decaying to the ground state as is the current paradigm. Moreover, we find that transitions between these multiply-excited states also contribute in the same narrow window around $13.5 \mathrm{~nm}$ as those originating from singly-excited states, and this striking property holds over a wide range of charge states. We thus reveal the doubly magic behavior of tin and the origins of the EUV light.

\footnotetext{
${ }^{1}$ Advanced Research Center for Nanolithography, Science Park 106, 1098 XG Amsterdam, The Netherlands. ${ }^{2}$ Department of Physics and Astronomy, and LaserLaB, Vrije Universiteit, De Boelelaan 1081, 1081 HV Amsterdam, The Netherlands. ${ }^{3}$ Keldysh Institute of Applied Mathematics, Miusskaya Square 4, 125047 Moscow, Russia. ${ }^{4}$ Industrial Focus Group XUV Optics, MESA+ Institute for Nanotechnology, University of Twente, Drienerlolaan 5, 7522 NB Enschede, The Netherlands. ${ }^{5}$ Zernike Institute for Advanced Materials, University of Groningen, Nijenborgh 4, 9747 AG Groningen, The Netherlands. ${ }^{6}$ Los Alamos National Laboratory, Los Alamos, NM 87545, USA. 凶email: o.versolato@arcnl.nl; jcolgan@lanl.gov
} 
$\mathrm{T}$ he complex, exotic electronic structure of highly charged ions of tin $(\mathrm{Sn})$ renders these ions of particular technological value as the enabler of next-generation nanolithography ${ }^{1-8}$. They are employed as emitters of photons in a narrow band closely matching the $2 \%$ reflection bandwidth centered at $13.5 \mathrm{~nm}$ of the most efficient multilayer optics ${ }^{9}$. This shortwavelength radiation is used to imprint smaller features on commercial microchips. The aptness of $\mathrm{Sn}$ ions to this application stems from their open- $4 d$-subshell structures ${ }^{10-20}$. Within these structures, $\Delta n=0$ one-electron-excited configurations are very well documented in the literature to decay to the ground state manifold via a multitude of transitions clustered together in unresolved transition arrays (UTAs) ${ }^{21}$, centered in the industrially relevant band around $13.5 \mathrm{~nm}$. Moreover, the average excitation energies of these configurations are similar across the isonuclear sequence $\mathrm{Sn}^{11+}-\mathrm{Sn}^{14+}$, making all these charge states excellent radiators of 13.5-nm light. In industrial applications, $\mathrm{Sn}$ ions are bred in laser-produced plasmas (LPPs) driven by a 10 - $\mu \mathrm{m}$-wavelength $\mathrm{CO}_{2}$-gas-laser cf. Fig. 1. Incorporation in EUV lithography of solid-state lasers, given the advances in their output power, appears promising. Switching to $1-\mu \mathrm{m}$-wavelength Nd:YAG lasers, for example, would be beneficial given the reduction of the required floor area and a strongly improved efficiency of converting electrical power to laser light, reducing carbon footprint. The tenfold decrease in laser wavelength $\lambda$ increases the critical plasma electron density $n_{\mathrm{c}}$ by two orders of magnitude, $n_{\mathrm{c}} \propto \lambda^{-2}$. This higher critical density causes EUV radiation to be created in plasma regions of higher density, and with overall larger optical depth ${ }^{22}$. Significant self-absorption of the emitted radiation in such dense, partially opaque plasma could lead to a broadening of the spectral emission out of the $2 \%$ bandwidth of interest, reducing efficiency. In the context of understanding and supporting the drive laser wavelength change in future industrial sources, calculation of complete and accurate opacity spectra and of the atomic data therein is essential for predictive simulations of source performance. These data are needed in radiation hydrodynamics $\operatorname{codes}^{23-26}$ and for the calculation of emission spectra. Without such accurate opacity data, the capability for predictive modeling would be severely impaired

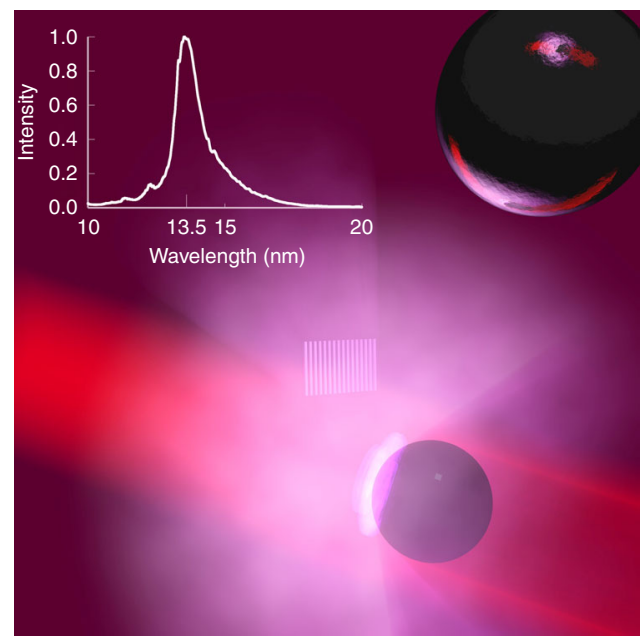

Fig. 1 Generation of extreme ultraviolet light. Laser-produced plasma based on the irradiation of tin ( $\mathrm{Sn}$ ) microdroplets by a high-energy nspulsed laser. This hot and dense plasma contains highly charged ions to generate extreme ultraviolet (EUV) light near $13.5 \mathrm{~nm}$ wavelength relevant for state-of-the-art nanolithography. A transmission grating spectrometer, set up under $\mathrm{a}-60^{\circ}$ angle with respect to the laser light propagation direction, enables unraveling the EUV spectrum. Figure by Tremani/ ARCNL. as, for example, modest underestimations of opacity could lead to significant underestimation of required drive laser intensities. The level of detail in the atomic structure necessary to ensure accurate simulations is an open question. In fact, long-standing discrepancies exist between the measurements of Sn opacity ${ }^{27}$ and various theoretical calculations using a variety of atomic structure and plasma codes $19,27,28$.

This paper identifies the main culprits of the historical discrepancies and addresses them, in order to generate reliable opacity spectra. These spectra are then shown to be in excellent agreement with the emission from a droplet-based EUV source. We find that EUV light predominantly originates from transitions between multiply-excited states. Contrary to the prevailing view, contributions from one-electron-excited states are minor. Moreover, we find that transitions between these multiply-excited states also strongly contribute to the same narrow $2 \%$ bandwidth around $13.5 \mathrm{~nm}$ as those originating from the well-known singlyexcited states. This serendipitous alignment of transitions furthermore occurs over a range of charge states $\mathrm{Sn}^{11+}-\mathrm{Sn}^{14+}$. A doubly magic behavior of tin is revealed. Having uncovered the true origins of the EUV light, our calculations will thus enable predictive modeling of future, more powerful and efficient laserdriven plasma sources of EUV light.

\section{Results}

Level structure of tin ions. The electronic energy level scheme presented in Fig. 2 exemplifies the characteristics in the atomic structure that need to be captured to accurately model a Sn plasma for nanolithograpic applications. This structure shows the average energies and widths of some of the typical configurations that play a role in the generation of EUV photons. The most notable phenomenon is that the excitation energies of electrons within the $n=4$ manifold shown are rather independent of the occupation of the manifold itself: the energy required to promote a $4 p$ electron to the $4 d$ subshell is almost the same regardless of the number of electrons in any of the other subshells. Fig. 2a shows that this holds either when changing charge state or excitation degree. This remarkable fact notwithstanding, the predominant view has been that only transitions of type $4 p^{6} 4 d^{m}-4 p^{6} 4 d^{m-1} 4 f+4 p^{5} 4 d^{m+1}$ (with $m=3 \ldots 0$ for $q=$ 11. . 14 in $\mathrm{Sn}^{q+}$ ), that is transitions from the singly-excited configurations significantly contribute to the emission of EUV photons. We will demonstrate the contrary: the contributions from multiply-excited states dominate. Taking as an example the $\mathrm{Sn}^{12+}$ ion, Fig. $2 \mathrm{~b}$ shows that the multiply-excited configurations, due to their large number of levels and high statistical weights (Fig. 2c), have large populations despite the lower excitation probabilities that are here described using a Boltzmann distribution. Surprisingly we find that, for example, the triply-excited states have similar partition function contributions as the singlyexcited states. These configurations therefore also must make an equally significant contribution to the production of EUV photons. The large number of decay channels is exemplified in Fig. $2 \mathrm{~d}$ with configurations decaying via electric dipole transitions towards the lower levels, which in turn decay again radiating similar energy photons. We note that Fig. 2 shows only a relatively small example of the number of configurations produced by successive $\Delta n=0$ excitations from the ground configuration. In the full calculations, presented in the next section, further $\Delta n=0$ excitations, and excitations into the $n=5$ shell, are also included. Many of these transitions also make significant contributions to the emission in the relevant wavelength range.

In the following, we present the opacity spectrum of a $\mathrm{Sn}$ plasma calculated in local thermodynamic equilibrium (LTE) at conditions relevant for the production of EUV light in an 
a

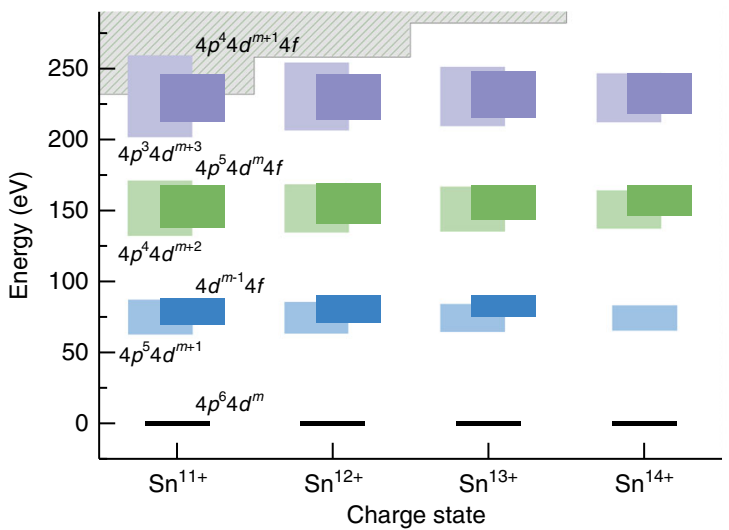

b

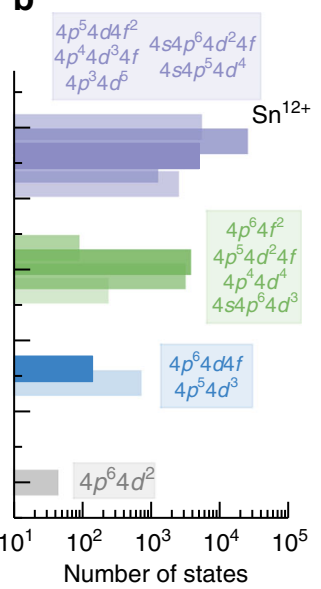

C

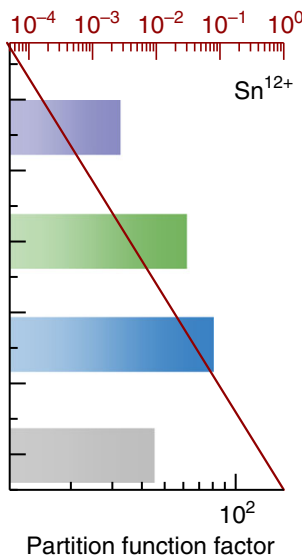

d

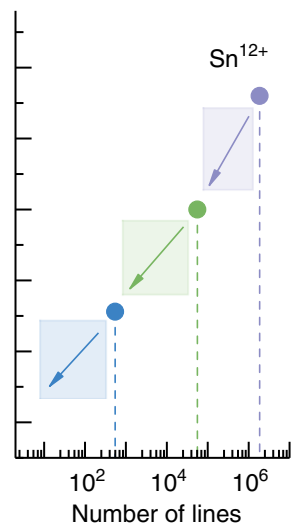

Fig. 2 Energy levels of tin ions and their population. a Schematic energy level diagram of the ions $S n^{11+}-S n^{14+}$, showing only selected $\Delta n=0$ transitions for clarity. The ground-state configurations of these ions take the form $4 d^{m}$, with $m=3-0$. The lowest-lying level of each ground-state manifold is shown in black and is fixed at an energy of $0 \mathrm{eV}$. The energy "spread" of an excited configuration is illustrated by a rectangle, centered at the average energy of the configuration and whose width represents the first moment of the level distributions. The shaded gray area denotes the ionization potentials of the ions. For the $\mathrm{Sn}^{12+}$ ion example case: $\mathbf{b}$ Statistical weight $\sum_{J}(2 J+1)$ per configuration. $\mathbf{c}$ Partition function factor, defined as $\sum_{i}\left(\exp \left(-E_{i} / k T\right) \sum_{J}(2 J+1)\right)$ in configurations $i$ grouped by excitation degree and (in red) exponential Maxwell-Boltzmann weighting factor exp $(-E / k T)$ with $T=32$ eV. $\mathbf{d}$ Number of lines per grouped configuration. In the final calculations, more excitations from further $\Delta n=0$ permutations and excitations into the $n=5$ shell are also considered, resulting in a total of $10^{10}$ dipole allowed transitions.

industrial setting. The calculations were performed using the Los Alamos code ATOMIC ${ }^{29,30}$, which takes as input state-of-the-art atomic data calculated with the Los Alamos suite of atomic $\operatorname{codes}^{31,32}$. The atomic structure was calculated using the semirelativistic Hartree-Fock approach implemented in the CATS code (based on Cowan's code ${ }^{33}$ ). These data are used by ATOMIC to calculate opacity spectra under the assumption of LTE with input from equation-of-state calculations performed with ChemEOS ${ }^{34,35}$, which ensures convergence of the partition function and thermodynamic consistency.

Atomic structure calculations. One of the most challenging aspects in calculations of Sn plasma opacities is the atomic structure of the highly charged Sn ions, due to their open $4 d$ subshells and the existence of strong configuration-interaction (CI) between levels in the $n=4$ manifold. The difficulties associated with accurate calculations of these level structures were presented in a recent study of Colgan et al. whereby $\mathrm{Sn}$ opacities, calculated using the aforementioned Los Alamos codes, were shown to be sensitive to the number of configurations included in the CI expansion ${ }^{19}$. However, without a suitable experimental benchmark, it was not possible to determine whether sufficient configurations were included in the atomic structure models.

In the current work, to ensure that the position of multiplyexcited levels and their oscillator strengths are calculated to the highest possible accuracy, full CI effects are taken into account for most of the single, double, and triple excitations of valence, $4 s$, and $4 p$ electrons of the ground-state configuration into the majority of $n=4$ and $n=5$ subshells. The list of configurations that were included in the full CI calculation for $\mathrm{Sn}^{12+}$ is presented in Table 1. This list comprises 94 configurations and generates over $3 \times 10^{5}$ fine-structure levels and more than $10^{10}$ dipoleallowed transitions, far more than were taken into account in other modeling work (see, for example, the work of Sasaki et al. ${ }^{36}$ on $\mathrm{CO}_{2}$-laser-driven $\mathrm{Sn}$ plasma). Similar sets of configurations were used for the neighboring ion stages. Moreover, we also included a significant number of other configurations for which the transitions were included using intermediate-coupling ${ }^{19}$. This mixed approach, called '2-mode', maintains the accuracy of CI

Table 1 Configuration list.

Inner subshells

\section{$4 s^{2} 4 p^{6}$}

$4 s^{2} 4 p^{5}$

$4 s^{2} 4 p^{4}$

$4 s^{1} 4 p^{6}$

$4 s^{2} 4 p^{3}$

$4 s^{1} 4 p^{5}$

$4 s^{2} 4 p^{6}$

$4 s^{2} 4 p^{5} 4 d$

List pertains to the configurations included in the full configuration interaction $(\mathrm{Cl})$ calculation for $\mathrm{Sn}^{12+}$ as used in the main text. Here, angular momenta $I=0-4 \rightarrow s-g$.

calculations for the most important transitions in the EUV regime, while retaining other levels that represent more highly excited states that are necessary for an accurate partition function and opacity at higher photon energies. Thus our approach meets the twin requirements of accuracy (for the crucial transitions), and completeness (for partition function convergence).

The list of configurations adopted for a given ion stage was determined by systematically increasing the number of configurations allowed to interact, and identifying for which configuration sets the positions of the dominant transitions converge. It is well-known that $\mathrm{ab}$ initio calculations performed in this manner do not necessarily reproduce experimental spectra to a high degree of accuracy. To circumvent this, it is standard practice in Cowan code calculations to introduce so-called scaling factors which pre-multiply the radial integrals appearing in the Hamiltonian matrix elements. As noted by Cowan ${ }^{33}$, these scaling factors account for the 'infinity of small perturbations' that are necessarily omitted in practical atomic structure calculations. Normally, a reduction of $10-15 \%$ of the radial integrals, that is, applying scaling factors of $0.85-0.9$, can bring theoretical calculations of level energies (and subsequently calculated transition wavelengths) into very good agreement with experimental observations. In our CATS calculations, the scaling factor 
is set to a standard 0.87 based on previous CATS calculations performed on a wide range of elements and charge states. When using the configuration set in Table 1, already much larger than previous calculations presented in ref. ${ }^{19}$, the position of the major transitions to the ground-state configuration (for example, $4 d^{2^{1}} \mathrm{G}_{4} \rightarrow 4 d 4 f^{1} \mathrm{H}_{5}$ in $\mathrm{Sn}^{12+}$ ) are in excellent agreement with the experimental observations in ref. ${ }^{14}$. Calculations were then performed using ATOMIC including atomic structure data for all relevant ion stages calculated in a manner similar to $\mathrm{Sn}^{12+}$. These calculations produced an intense emission feature in good qualitative agreement with the measured spectrum, apart from a crucial shift in the central position of the emission feature towards shorter wavelength. In fact, the feature was positioned outside the relevant $2 \%$ emission band for nanolithography. Since it was established that the well-known transitions to the ground manifold are correctly calculated, the discrepancy must originate from inaccurate positioning of transitions between excited states.

This unexpected finding led us to re-consider our atomic structure calculations for the excited-excited transitions. Little data for such excited transitions are available for the $\mathrm{Sn}^{11+}-\mathrm{Sn}^{14+}$ ions. For $\mathrm{Sn}^{14+}$, the calculated electronic structure differed from the interpretation of charge-exchange measurements (and accompanying calculations) of D'Arcy et al. ${ }^{37}$ by around $2 \%$ in wavelength position. We found that reducing the scaling factors in CATS to 0.75 yielded much better agreement with these data and with the experimental charge-exchange emission spectra of Ohashi et al. ${ }^{17}$. This further reduction may account for greater correlation effects between these high-energy configurations, arising from their high density of states.

Adopting scaling factors of 0.87 and 0.75 for the transitions to the ground manifolds and for transitions between excited states, respectively, opacity spectra are calculated at the representative temperature and density of $32 \mathrm{eV}$ and $0.002 \mathrm{~g} \mathrm{~cm}^{-3}\left(\sim 10^{20} \mathrm{e}^{-} \mathrm{cm}^{-3}\right)$. These plasma conditions, used throughout this paper, are typical for a $1-\mu \mathrm{m}$-driven LPP tailored for emission of 13.5-nm photons, as suggested by radiation hydrodynamic simulations ${ }^{24,25}$ (see "Methods" section). Light emission is described as occurring at sub-critical density, close to the sonic surface of the ablation front at an electron density of several $10^{20} \mathrm{e}^{-} \mathrm{cm}^{-3}$. These density and temperature values also support the LTE approach adopted (see Methods).

In Fig. 3, the contribution of the four ion stages $\mathrm{Sn}^{11+}-\mathrm{Sn}^{14+}$ to the total opacity is shown. Each spectrum shows the three major bound-bound contributions, which can be loosely associated with singly-excited, doubly-excited, and triply-excited states (see Fig. 2). Remarkably, for this choice of temperature and density, the well-known transitions to the ground levels comprise only $11 \%$ of the total opacity in the $5-20 \mathrm{~nm}$ range. The remaining $89 \%$ is associated with higher-lying transitions: $26 \%$ is attributed to transitions between singly- and doubly-excited states, $25 \%$ to transitions between doubly- and triply-excited states, and $38 \%$ is associated with higher excitations. Even when considering the opacity in a $2 \%$ bandwidth around $13.5 \mathrm{~nm}$, the transitions from singly-excited configurations only account for $19 \%$ of the total opacity.

Comparison to experiment. In order to benchmark our present calculations, we have made comparisons with experimental laserproduced tin-plasma spectra recorded for a variety of laser intensities, which determine the plasma properties such as temperature and degree of ionization ${ }^{38}$. Guided by our radiationhydrodynamics simulations (see below), we performed ATOMIC calculations at predicted ranges of plasma temperatures and densities, and compared these to the measured spectra to ascertain the specific sets of conditions that lead to best agreement between modeling and experiment. The experimental spectra
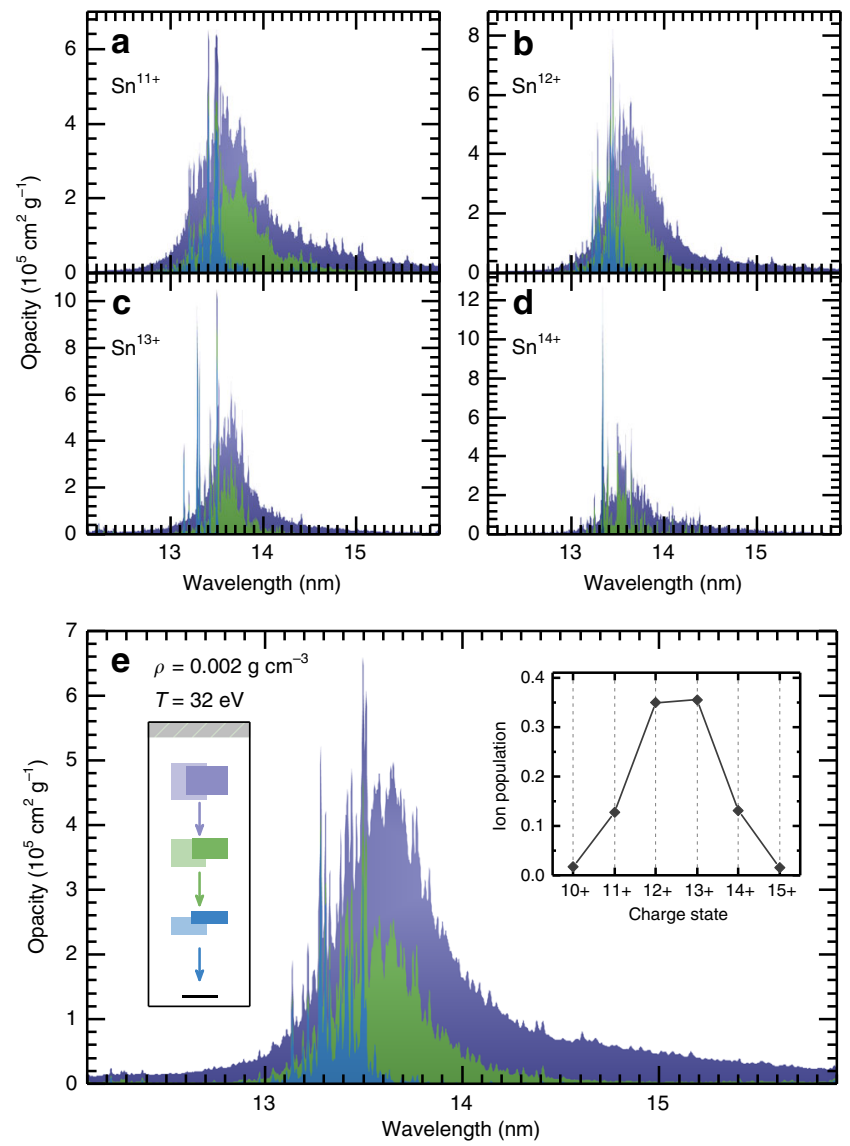

Fig. 3 Opacity spectra of tin ions. Calculations were performed for a $32 \mathrm{eV}$, $0.002 \mathrm{~g} \mathrm{~cm}^{-3} \mathrm{Sn}$ plasma in local thermodynamic equilibrium. The shaded areas represent the cumulative contributions (that is the next contribution is plotted stacked on top of the previous one) stemming from different types of excited states. They are divided according to the energy of the lower state into which the ions radiatively decay: in blue, transitions into the ground state manifold (from single-electron excited states); in green, transitions into levels with energies between 0 and $150 \mathrm{eV}$ (comprising mainly transitions between singly- and doubly-excited states); in purple, transitions occurring between doubly-excited states (lying above $150 \mathrm{eV}$ ) and higher-lying multiply-excited states. a-d Opacity spectra of the individual Sn ions. e Total opacity spectrum. The left inset contains the simplified atomic structure of $\mathrm{Sn}^{13+}$ (see Fig. 2 and main text), while the right inset shows the relative charge state population of the plasma. All spectra are convoluted with a Gaussian profile to improve the visibility of the various contributions.

were obtained by irradiating a molten Sn microdroplet, $30 \mu \mathrm{m}$ in diameter, with a 15 -ns-long Nd:YAG laser pulse having a flat-top spatial profile of $96 \mu \mathrm{m}$ diameter ${ }^{38,39}$. The emission in the EUV regime is recorded using a wavelength-calibrated spectrometer ${ }^{40}$. The experiment is described in further detail in ref. ${ }^{38}$. Spectra have been recorded at three distinct laser intensities: $1.4 \times 10^{11} \mathrm{~W}$ $\mathrm{cm}^{-2}$ (this value gives optimal performance with respect to EUV emission $^{38}$ ), $6.6 \times 10^{10} \mathrm{~W} \mathrm{~cm}^{-2}$, and $3.9 \times 10^{10} \mathrm{~W} \mathrm{~cm}^{-2}$.

To enable a comparison between opacity calculations and experimental emission spectra, we must adopt a model for radiation transport through the plasma medium. In LTE, the complexity of the radiation transfer problem is reduced since the (spectral) emissivity $\eta_{\lambda}$ and the (spectral) opacity $\kappa_{\lambda}$ are linked by the relation $\eta_{\lambda}=B_{\lambda} \cdot \kappa_{\lambda} \cdot \rho^{41}$, where $B_{\lambda}$ is Planck's spectral radiance and $\rho$ the mass density (the product of $\kappa_{\lambda}$ and $\rho$ being the absorptivity $\alpha_{\lambda}$ ). Generally, even in the $1 \mathrm{D}$ approximation, the radiation transport equation should be solved numerically 
along the plasma column leading to the observer. This would necessitate the calculation of opacities for each $(\rho, T)$ pair. Such an endeavor, particularly in view of the level of detail in the opacity calculations here presented, is beyond the scope of this paper. Instead, a single-temperature, single-density approach is here employed. Indeed, recent experiments by Schupp et al. ${ }^{22}$ have indicated that a dominant fraction of the EUV emission may be produced in such a quasi-stationary ${ }^{24}$ single-density, singletemperature region. For such a medium, the spectral flux $I_{\lambda}$ can be determined using the simple solution $I_{\lambda}=B_{\lambda}[1-\exp (-\tau)]$, with the optical depth $\tau$ defined as the product between $\alpha_{\lambda}$ and the transport path-length $L$. The temperature of the opacities are chosen such that the calculated charge state contributions matched the observed one. In order to justify the choice of density and path length, we have undertaken radiation hydrodynamic simulations using the RALEF-2D code $24,25,42$. These simulations indicate that the vast majority of the emission originates in a 10- to $30-\mu \mathrm{m}$-thick plasma having density on the order of $10^{20} \mathrm{e}^{-} \mathrm{cm}^{-3}$, rather independent of laser intensity (see Methods section). In our comparisons below, we use a constant $30-\mu \mathrm{m}$ path length at $10^{20} \mathrm{e}^{-} \mathrm{cm}^{-3}$ density.

In Fig. 4, a comparison between the experimental emission spectra and the spectral fluxes obtained from applying the aforementioned 1D radiation transport model to the ATOMIC opacity calculations is presented for three different laser intensities. Overall, the level of agreement is excellent. Fig. 4a shows the spectrum for the laser intensity $1.4 \times 10^{11} \mathrm{~W} \mathrm{~cm}^{-2}$. The spectral flux calculated using the single-density, singletemperature approach is able to reproduce the experimental emission strikingly well. The figure also shows the plasma opacity from Fig. 3, which makes apparent that without the contributions from the multiply excited states it would not be possible to fully explain the experimental spectrum. To further highlight the importance of these transitions, our results are compared with calculations from previous works. The dashed line was obtained using the opacity from Colgan et al. ${ }^{19}$, which, as discussed in a previous section, perfectly exemplifies the shift of the main emission feature towards shorter wavelengths, arising from inaccuracies in the calculated line positions for transitions between multiply-excited states. The dotted line is based on opacity data from ref. ${ }^{27}$, generated with the HULLAC code at an electron temperature of $30 \mathrm{eV}$ but at a higher mass density of $0.01 \mathrm{~g} \mathrm{~cm}^{-3}$. To enable the comparison at similar optical depth, the path length used to calculate the spectral flux was five times shorter. These calculations significantly overestimate the width of the main emission feature and are in poor agreement with the experimental spectra and, while some disparities could be explained by the density difference, the overall discrepancy may be attributed to the atomic structure employed.

Figure $4 \mathrm{~b}$ and $\mathrm{c}$ show the comparison between calculations and experiment at lower laser intensities. These spectra clearly exhibit the spectral signature of lower $\mathrm{Sn}$ charge states. The data are still in good agreement, even though some deviations are observed in the $14-16 \mathrm{~nm}$ region. Indeed, our assumptions might (partially) break down at lower intensities, due to inapplicability of the single-density, single-temperature approach or deviation from the calculated charge state balance. The opacity breakdowns for these two cases show that the relevance of the multiply-excited states decreases for the lower plasma temperatures but they are still necessary for complete opacity spectra.

\section{Discussion}

It is interesting to consider why multiply-excited states appear to be so important in Sn plasma. Lower Z elements under similar

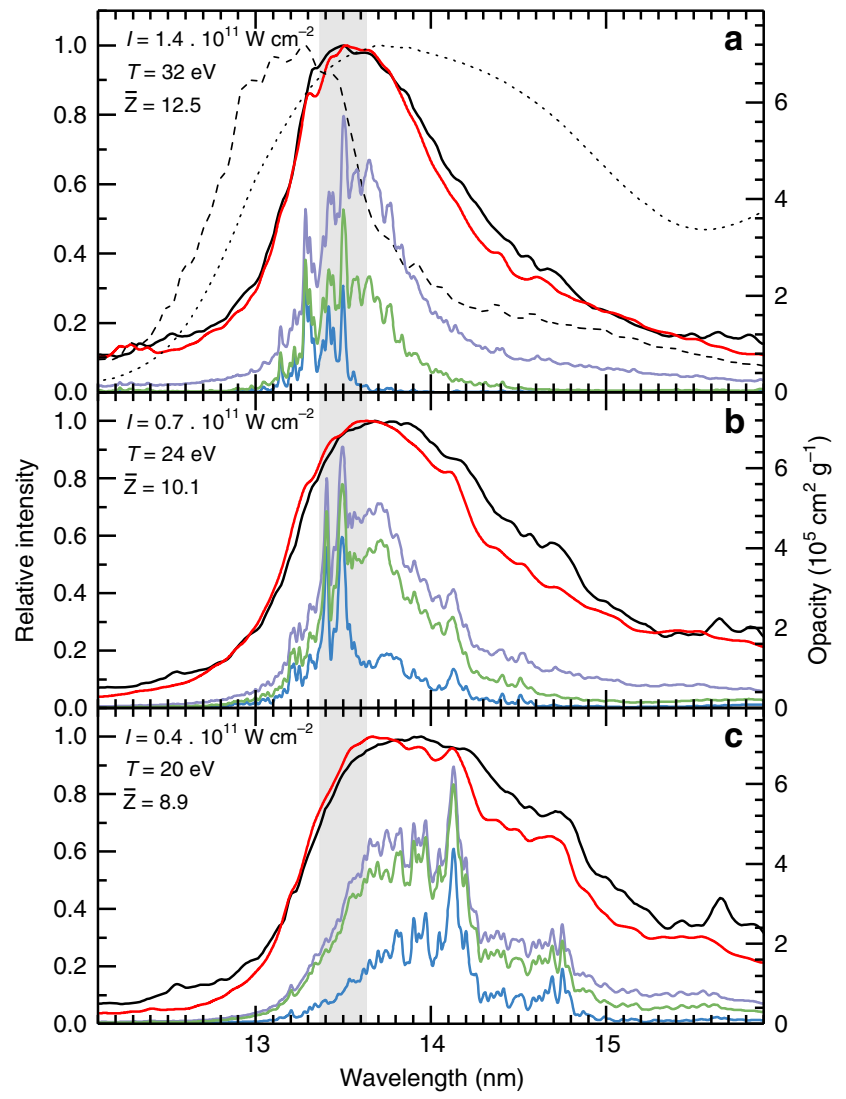

Fig. 4 Comparing calculation to experiment. Experimental spectra (black solid lines) and calculated fluxes (red solid lines) are shown, normalized to their respective maximum. The spectral fluxes are the result of the 1D radiation transport through a single-density $\left(0.002 \mathrm{~g} \mathrm{~cm}^{-3}\right)$, single-

temperature plasma (see main text). a Spectral flux calculated using an opacity spectrum from ref. ${ }^{19}$ (dashed line) and the spectral flux obtained from HULLAC calculations ${ }^{27}$ (dotted line). Opacity spectra, broken down according to the various contributions illustrated in Fig. 3 are also shown. The mean charge state $\bar{Z}$ of the calculation is given as well. The shaded gray area highlights the industrially-relevant $2 \%$ bandwidth around $13.5 \mathrm{~nm}$. b, c Same as in a but for two lower laser intensities and associated plasma temperatures.

conditions, for example, $\mathrm{Al}$ or $\mathrm{Fe}$, are also ionized approximately ten times; however, this results in ion stages with much simpler configurations, such as open-2p-or open-3p-subshells in $\mathrm{Al}$ and Fe respectively. Multiply-excited states in these subshells have much smaller statistical weights compared to the multiply-excited states of $\mathrm{Sn}$, and so their relative contribution to the plasma emission is also much smaller. If instead one looks once again at open- $4 d$-subshells but in a lighter element, such as neutral Sr, the multiply-excited states are energetically much further away from the ground state due to the smaller nuclear charge. Compared to $\mathrm{Sn}$, this significantly reduces their contribution to the partition function. For example, the $4 p^{5} 4 d^{3}$ configuration in neutral Sr at a temperature of $1 \mathrm{eV}$ contributes $10^{-13}$ times less than in the case of the same configuration for $\mathrm{Sn}$ ions in a $30 \mathrm{eV}$ plasma. For all of these reasons, Sn finds itself in this peculiar position in which, due to the plasma conditions necessary for nanolithography, the complicated structures of these multiple, $n=4$ excited-electron configurations play a staggeringly important role. These multiplyexcited states will also play an important role in other shortwavelength applications ${ }^{43}$ ranging from beyond-EUV lithography $^{2}$ to water-window imaging ${ }^{4}$ where candidate elements for LPP exhibit strong $n=4-4$ transition arrays that contribute 

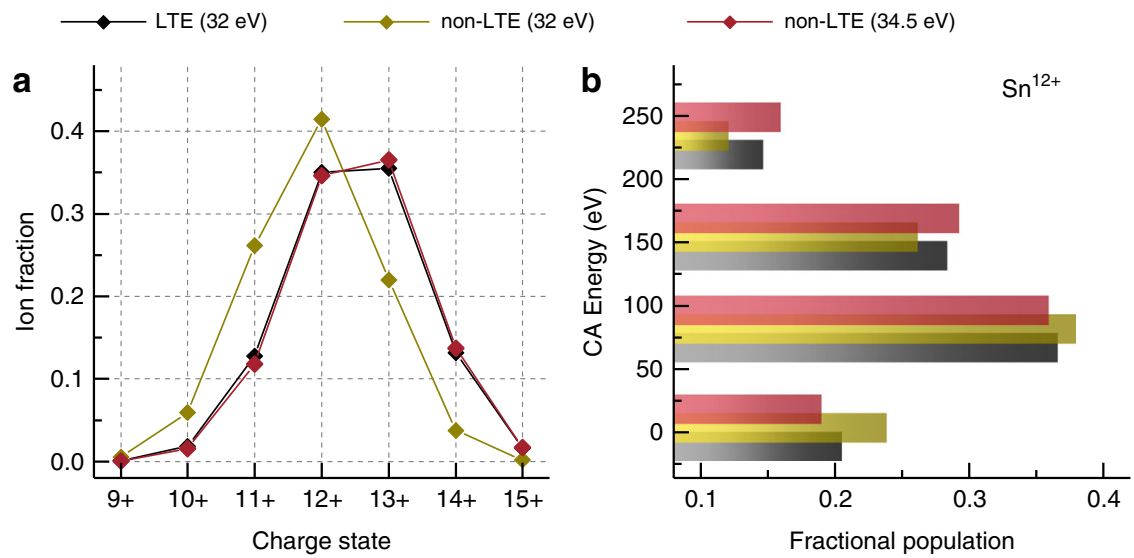

Fig. 5 Validity of local thermodynamic equilibrium (LTE). a Charge state distribution calculations for a $32 \mathrm{eV} 0.002 \mathrm{~g} \mathrm{~cm}^{-3}\left(\sim 1 \times 10^{20} \mathrm{e}^{-} \mathrm{cm}^{-3}\right)$ plasma under both LTE and non-LTE conditions; a non-LTE calculation for a $34.5 \mathrm{eV}$ temperature is additionally shown. $\mathbf{b}$ Fractional population in configurations grouped by excitation degree (as in Fig. 2) under conditions indicated in a from configuration-averaged (CA) calculations (see main text). CA energies are offset for better visibility.

to radiation, with emission wavelength decreasing with the atomic number following a quasi-Moseley law ${ }^{45}$.

In conclusion, we show that, contrary to the prevailing view, the opacity of high-density Sn plasma of relevance for nanolithographic applications are characterized by a remarkably large contribution from multiply-excited states. Multiple electron excitation into the $4 d$ or $4 f$ subshells leads to states with very high angular momenta and large statistical weights. These configurations are heavily affected by configuration-interaction, making them challenging to calculate accurately. Crucially, the dominant bound-bound transitions involving these multiply-excited states are clustered close to $13.5 \mathrm{~nm}$ wavelength, as are the transitions from singly-excited states. These insights finally enable explaining the intense emission feature from Sn LPPs that are right now entering high-volume manufacturing in the nanolithographic industry for the continued progress of miniaturization of semiconductor devices. The calculations are shown to be in excellent agreement with experimental emission spectra from a dropletbased, Sn laser-produced-plasma source of EUV light. Our results will enable accurate simulation of emission spectra from radiation-hydrodynamic simulations of high-density Sn plasmas aiding the development of future more powerful and energyefficient EUV light sources.

\section{Methods}

Los Alamos atomic codes. The atomic structure calculations used in our modeling are discussed in detail in the main text. These calculations are augmented by photoionization cross sections computed from the Los Alamos GIPPER code ${ }^{32}$. These data are read into the plasma modeling code ATOMIC. In its LTE mode, ATOMIC computes the partition function for a given temperature \& density using the CHEMEOS ${ }^{34,35}$ option, which computes equation-of-state quantities in a chemical picture. ATOMIC then computes the resulting plasma opacity or emissivity using this partition function, coupled with the detailed atomic transition data from CATS.

Validity of local thermodynamic equilibrium. The large number of available $\left(\sim 10^{10}\right)$ transitions computationally precludes performing full-detail collisional radiative modeling. It is common to invoke local thermodynamic equilibrium (LTE) in such cases to enable the prediction of experimental spectra using Boltzmann-distributed excitation-population densities. Several validity criteria for LTE are available, mostly revised versions of the original proposed by $\mathrm{Griem}^{46}$, for atomic systems of limited complexity. These validity criteria would indicate establishment of LTE at a density scale of $10^{20}-10^{21} \mathrm{e}^{-} \mathrm{cm}^{-3}$, supporting its invocation in the current work.

To enable a more direct validation, configuration-averaged non-local thermodynamic equilibrium (non-LTE) calculations were also run using ATOMIC in its non-LTE mode. The configuration-average atomic data needed for the nonLTE calculations were generated using the LANL suite of atomic physics codes. The CATS calculations, in this case, were made with the default Cowan scale parameters. Rates of collisional excitation and ionization, and autoionization (and the inverse of these processes) are needed to perform a non-LTE calculation. The generation of these data, plus the need for a full matrix solve of the collisionalradiative problem, means that we normally require about three orders of magnitude more computing resources than a LTE calculation that uses the same number of atomic states. This is why we considered the much simplified configuration-average problem with a small number of configurations, taking into account only $\Delta n=0,1$ excitations but including the doubly and triply excitations within the $n=4$ manifold. The calculations, shown in Fig. 5, were performed for a $32 \mathrm{eV}, 0.002 \mathrm{~g} \mathrm{~cm}^{-3}\left(\sim 1 \times 10^{20} \mathrm{e}^{-} \mathrm{cm}^{-3}\right)$ plasma under both LTE and non-LTE conditions. It is immediately apparent that there is difference in the charge state distribution and the obtained non-LTE spectrum would not be relevant for the comparison at the given temperature. Still, a large fraction of the population is shown to be retained in the multiply-excited configurations. For a fairer comparison, following the established approach in Sasaki et al. ${ }^{36}$ (also see the review by Bauche et al. ${ }^{47}$ ) we slightly increase the non-LTE temperature to $34.5 \mathrm{eV}$ such that the mean charge state $\bar{Z}$ in non-LTE equals that of the $32 \mathrm{eV}-\mathrm{LTE}$ calculations. This small step in temperature renders the charge state distribution indistinguishable from the LTE one (see Fig. 5). The small change in the temperature needed to reproduce the LTE calculations furthermore strongly indicates that the system is close to LTE at $32 \mathrm{eV}$ and $0.002 \mathrm{~g} \mathrm{~cm}^{-3}$. Photoexcitation by the radiation field, not included in our non-LTE calculations, would bring the system even closer to LTE. At these temperatures, our non-LTE calculations would indicate similarly strong contributions as in LTE of the multiply-excited states to the emission of EUV light from tin LPP.

Radiation-hydrodynamics simulations. We have performed RALEF-2D simulations to explore where the extreme ultraviolet light is generated in a laserproduced-plasma resulting from the irradiation of a Sn microdroplet by a highintensity, $1-\mu \mathrm{m}$-wavelength laser-pulse. RALEF-2D is a two-dimensional numerical code which solves the 2D single-fluid, one-temperature hydrodynamics equations and the spectral radiation transfer equation, using opacity tables generated with the THERMOS code ${ }^{48}$. In the RALEF-2D code, energy transport via radiation is coupled directly to the fluid through the fluid energy equation, and therefore spectral radiation transport is treated in a self-consistent manner. As has been demonstrated previously, the radiation-hydrodynamic approach implemented in RALEF-2D makes it very apt in simulating systems in which energy transport by thermal radiation plays a significant role $24,25,42,49$. The code was also recently validated against measurements of laser-induced propulsion of Sn microdroplets ${ }^{50}$

The simulations begin by setting the initial conditions of the system: droplet size, spatial and temporal laser profiles, and laser energy. For the three spectra shown in Fig. 4, the parameters are as follows: $30 \mu \mathrm{m}$ droplet diameter; box-shaped laser profiles, $96 \mu \mathrm{m}$ spatially, and $15 \mathrm{~ns}$ temporally; laser energies of $170 \mathrm{~mJ}, 78 \mathrm{~mJ}$ and $47 \mathrm{~mJ}$, corresponding to intensities of $1.4 \times 10^{11} \mathrm{~W} \mathrm{~cm}^{-2}, 0.7 \times 10^{11} \mathrm{~W} \mathrm{~cm}-2$ and $0.4 \times 10^{11} \mathrm{~W} \mathrm{~cm}^{-2}$. From the simulation results, it is possible to obtain twodimensional spatial profiles of temperature and density as a function of time. To simplify the analysis, we have generated one-dimensional profiles of these two quantities along a $-60^{\circ}$ line-out with respect to the laser propagation direction, effectively emulating the observation angle of the spectrometer in the experiment. The duration of the laser beam is sufficient to have a steady-state ablation front, and therefore in the following we will look at the time instant at the end of the laser pulse (before it is turned off).

In Fig. 6a, we plot the spatial variation of temperature and density obtained from the RALEF-2D code along the aforementioned $-60^{\circ}$ line-out for the three relevant laser power densities. The spatial variation of these two quantities sets the 


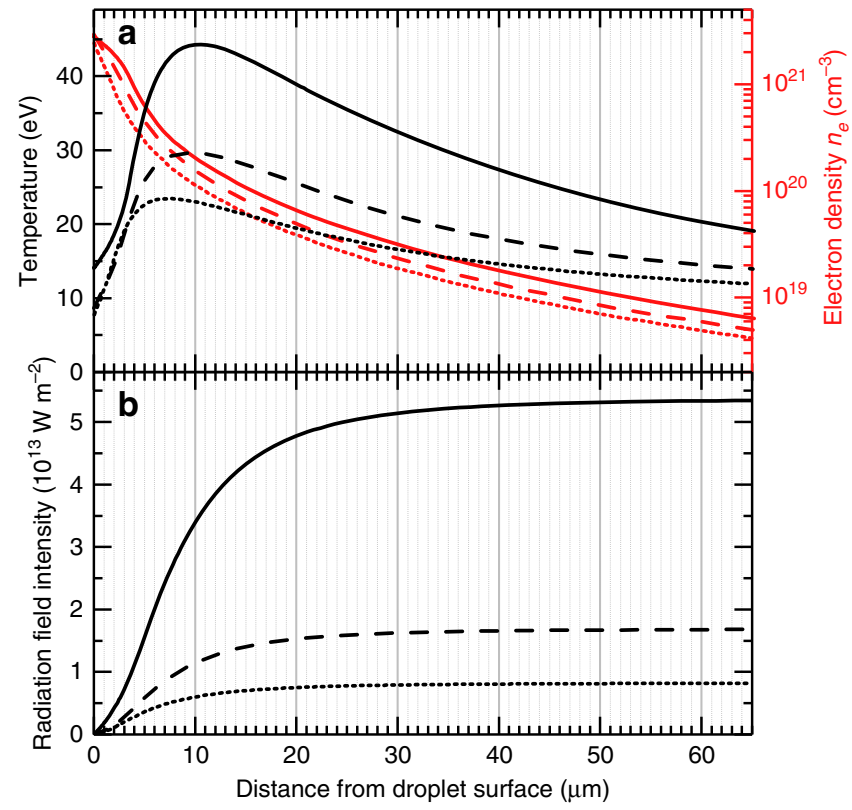

Fig. 6 Simulation results. a Temperature and density profiles calculated by RALEF-2D simulations for laser intensities of 0.4 (dotted), 0.7 (dashed) and 1.4 (solid) $\times 10^{11} \mathrm{~W} \mathrm{~cm}^{-2}$, respectively. b Radiation field intensity obtained by solving the simplified frequency-integrated transport equation, from Eq. (4), along the density and temperature profiles in $\mathbf{a}$.

radiation properties of the plasma medium. In order to pinpoint the origin of the intense EUV emission in the simulated plasma, we have undertaken a postsimulation analytic study of radiation transport using these temperature and density profiles. In our simplified approach, we first assume that the effects of scattering are small relative to absorption mechanisms; secondly, we consider frequency-integrated variables to simplify the amount of data necessary for these calculations. In this static limit, all variables are ultimately a function of only the position along the transport length $s$ and therefore the radiation transport equation reads:

$$
\frac{\partial I}{\partial s}=\alpha[B(T)-I]
$$

with $I$ the frequency-integrated radiation intensity, $s$ the path length variable, $\alpha$ the non-linearly averaged absorption coefficient, and $B(T)=\sigma T^{4} / \pi$. In order to solve the previous equation, one needs an approximate value for $\alpha$. In general, this quantity is equated to the Planck mean opacity:

$$
\alpha \approx \alpha_{P} \equiv \frac{1}{B(T)} \int_{0}^{\infty} \alpha_{\nu} B_{\nu} \mathrm{d} \nu
$$

The Planck mean opacity, in the case of Sn plasma, can be calculated as follows ${ }^{24}$ :

$$
\alpha_{P}\left[\mathrm{~m}^{-1}\right]=3.3 \cdot 10^{-7} \cdot \rho\left[\mathrm{g} \mathrm{cm}^{-3}\right] \cdot \mathrm{T}^{-1}[\mathrm{eV}] .
$$

This equation allows for the calculation of the spatial variation of $\alpha_{P}$, denoted $\alpha(s)$ in the following, using the temperature and density profiles shown in Fig. 6a. Although this approach is indeed a simplified version of that taken in the RALEF2D code (which explicitly employs density- and temperature-dependent spectral absorption coefficients $\alpha_{v}(\rho, T)$ calculated using the THERMOS code), it can be used to identify the position and extent of the EUV-emissive zone. The solution to Eq. (1) reads:

$$
\begin{aligned}
I(s)= & I_{0} \exp \left[-\int_{s 0}^{s} \alpha\left(s^{\prime}\right) \mathrm{d} s^{\prime}\right] \\
& +\int_{s 0}^{s} \alpha\left(s^{\prime}\right) B\left(s^{\prime}\right) \exp \left[-\int_{s^{\prime}}^{s} \alpha\left(s^{\prime \prime}\right) \mathrm{ds}^{\prime \prime}\right] \mathrm{d} s^{\prime},
\end{aligned}
$$

which can be easily solved numerically using the line-out profiles obtained from RALEF-2D.

The solution to $I(s)$ is presented, alongside the temperature and density profiles, for the three laser intensities in Fig. 6. These profiles, besides their absolute values, are rather independent of laser intensity. The laser light is found to be dominantly absorbed in the underdense corona before reaching the critical surface, in line with the findings in ref. ${ }^{24}$. The profiles in panel b clearly show that the vast majority of the radiation field intensity builds up in the first $20 \mu \mathrm{m}$, then levelling off as the lower temperature, rarefied plasma does not contribute strongly to the radiation field, neither in emission nor in absorption. In all three cases explored, half of the far-field radiation field intensity is shown (see Fig. 6b) to be achieved at an electron density of $3 \times 10^{20} \mathrm{~cm}^{-3}$ and a $80 \%$ fraction of the final radiation field intensity is built up over a $10 \mu \mathrm{m}$ path length. These values found are in good agreement with the ones chosen in the $1 \mathrm{D}$ radiation model necessary to compare the opacity calculations to the experimental emission spectra, where a path length of $30 \mu \mathrm{m}$ together with the density of $10^{20} \mathrm{~cm}^{-3}$ gives very good agreement with the experimental data. At an electron density of $3 \times 10^{20} \mathrm{~cm}^{-3}$, the plasma is even closer to LTE and the slightly higher temperature, cf. Fig. 6 , at this higher density will result in even larger population fractions in the multiply-excited states given their exponential dependence on the plasma temperature.

On the other hand, temperature peak values given by the code are higher than expected. These results are rather inconsistent with our spectroscopic measurements simply on the basis of charge state balance. If we look at the highest intensity case in Fig. 6, temperatures over $45 \mathrm{eV}$ are observed. At this temperature, we would expect a plasma average charge state above $14+$ according to ref. ${ }^{24}$. This is demonstrably not the case. These discrepancies could be originating from the opacity tables employed in RALEF-2D, which do not include the contribution from multiply-excited states as outlined in the present work, and underline the importance of obtaining accurate opacity tables. Some ambiguity about the plasma temperature which best matches the data remains. As the laser intensities and associated temperatures are shown to have a minor influence on density and length scale results (cf. Fig. 6), these minor inconsistencies do not impact the results of said density and length scales.

\section{Data availability}

The data that support the findings of this study are available from the corresponding authors upon reasonable request.

\section{Code availability}

RALEF-2D is available upon reasonable request from M.M. Basko. Correspondence and requests for ATOMIC and related codes used in the paper should be addressed to J. Colgan.

Received: 28 October 2019; Accepted: 23 March 2020; Published online: 11 May 2020

\section{References}

1. Versolato, O. O. Physics of laser-driven tin plasma sources of EUV radiation for nanolithography. Plasma Sources Sci. Technol. 28, 083001 (2019).

2. Tallents, G., Wagenaars, E. \& Pert, G. Optical lithography: lithography at EUV wavelengths. Nat. Photonics 4, 809-811 (2010).

3. Wagner, C. \& Harned, N. EUV lithography: lithography gets extreme. Nat. Photonics 4, 24-26 (2010).

4. Waldrop, M. M. The chips are down for Moore's law. Nat. News 530, 144 (2016).

5. Banine, V. Y., Koshelev, K. N. \& Swinkels, G. H. P. M. Physical processes in EUV sources for microlithography. J. Phys. D: Appl. Phys. 44, 253001 (2011).

6. Fomenkov, I. et al. Light sources for high-volume manufacturing EUV lithography: technology, performance, and power scaling. Adv. Opt. Techn. 6 173-186 (2017).

7. Kawasuji, Y. et al. Key components technology update of the $250 \mathrm{~W}$ high-power LPP-EUV light source. SPIE Adv. Lithogr. 10143, 101432G-101432G (2017).

8. Purvis, M. et al. In Extreme Ultraviolet (EUV) Lithography IX, vol. 10583 476-485 (International Society for Optics and Photonics, 2018)

9. Huang, Q. et al. Spectral tailoring of nanoscale EUV and soft x-ray multilayer optics. Appl. Phys. Rev. 4, 011104 (2017).

10. Azarov, V. I. \& Joshi, Y. N. Analysis of the $4 \mathrm{~d}^{7}-4 \mathrm{~d}^{6} 5 \mathrm{p}$ transition array of the eighth spectrum of tin: Sn VIII. J. Phys. B: . Mol. Opt. Phys. 26, 3495-3514 (1993).

11. Svendsen, W. \& O'Sullivan, G. Statistics and characteristics of xuv transition arrays from laser-produced plasmas of the elements tin through iodine. Phys. Rev. A 50, 3710-3718 (1994).

12. Churilov, S. S. \& Ryabtsev, A. N. Analyses of the Sn IX-Sn XII spectra in the EUV region. Phys. Scr. 73, 614-619 (2006).

13. Churilov, S. S. \& Ryabtsev, A. N. Analysis of the $4 \mathrm{p}^{6} 4 \mathrm{~d}^{7}-\left(4 \mathrm{p}^{6} 4 \mathrm{~d}^{6} 4 \mathrm{f}+4 \mathrm{p}^{5}\right.$ $\left.4 \mathrm{~d}^{8}\right)$ transitions in the Sn VIII spectrum. Opt. Spectrosc. 100, 660-666 (2006).

14. Churilov, S. S. \& Ryabtsev, A. N. Analysis of the spectra of In XII-XIV and Sn XIII-XV in the far-VUV region. Opt. Spectrosc. 101, 169-178 (2006).

15. Ryabtsev, A. N., Kononov, É. Y. \& Churilov, S. S. Spectra of rubidium-like Pd X-Sn XIV ions. Opt. Spectrosc. 105, 844-850 (2008).

16. Tolstikhina, I. Y., Churilov, S. S., Ryabtsev, A. N. \& Koshelev, K. N. In EUV Sources for Lithography (ed. Bakshi, V.) chap. 4, 113-148 (SPIE Press, 2006).

17. Ohashi, H. et al. EUV emission spectra in collisions of multiply charged Sn ions with He and Xe. J. Phys. B. . Mol. Opt. Phys. 43, 065204 (2010). 
18. Windberger, A. et al. Analysis of the fine structure of $\mathrm{Sn}^{11+}-\mathrm{Sn}^{14+}$ ions by optical spectroscopy in an electron-beam ion trap. Phys. Rev. A 94, 012506 (2016).

19. Colgan, J. et al. Atomic structure considerations for the low-temperature opacity of Sn. High. Energy Density Phys. 23, 133-137 (2017).

20. Torretti, F. et al. Optical spectroscopy of complex open- $4 d$-shell ions $\mathrm{Sn}^{7+}-\mathrm{Sn}^{10+}$. Phys. Rev. A 95, 042503 (2017).

21. Bauche, J., Bauche-Arnoult, C. \& Klapisch, M. Transition arrays in the spectra of ionized atoms. Adv. At. Mol. Phys. 23, 131-195 (1988).

22. Schupp, R. et al. Radiation transport and scaling of optical depth in Nd:YAG laser-produced microdroplet-tin plasma. Appl. Phys. Lett. 115, 124101 (2019).

23. Scott, H. A. Cretin - a radiative transfer capability for laboratory plasmas. $J$. Quant. Spectrosc. Radiat. Transf. 71, 689-701 (2001).

24. Basko, M. M., Novikov, V. G. \& Grushin, A. S. On the structure of quasistationary laser ablation fronts in strongly radiating plasmas. Phys. Plasmas 22, 053111 (2015).

25. Basko, M. On the maximum conversion efficiency into the 13.5-nm extreme ultraviolet emission under a steady-state laser ablation of tin microspheres. Phys. Plasmas 23, 083114 (2016).

26. $\mathrm{Su}, \mathrm{M}$. et al. Evolution analysis of EUV radiation from laser-produced tin plasmas based on a radiation hydrodynamics model. Sci. Rep. 7, 45212 (2017).

27. Fujioka, S. et al. Opacity effect on extreme ultraviolet radiation from laserproduced tin plasmas. Phys. Rev. Lett. 95, 235004 (2005).

28. Zeng, J., Gao, C. \& Yuan, J. Detailed investigations on radiative opacity and emissivity of tin plasmas in the extreme-ultraviolet region. Phys. Rev. E 82, 026409 (2010).

29. Magee, N. H. et al. In AIP Conf. Proc., vol. 730, 168-179 (AIP, 2004)

30. Hakel, P. et al. The new Los Alamos opacity code ATOMIC. J. Quant. Spectr. Rad. Transf. 99, 265-271 (2006).

31. Abdallah, J. Jr., Clark, R., Peek, J. \& Fontes, C. Kinetics calculations for near Ne-like ions. J. Quant. Spectr. Rad. Transf. 51, 1-8 (1994).

32. Fontes, C. et al. The Los Alamos suite of relativistic atomic physics codes. $J$. Phys. B: . Mol. Opt. Phys. 48, 144014 (2015).

33. Cowan, R. D. The Theory of Atomic Structure and Spectra (University of California Press, 1981).

34. Hakel, P. \& Kilcrease, D. P. In AIP Conf. Proc., vol. 730, 190-199 (AIP, 2004).

35. Kilcrease, D., Colgan, J., Hakel, P., Fontes, C. \& Sherrill, M. In Workshop on Astrophysical Opacities, vol. 515 of Astronomical Society of the Pacific Conference Series (ASP, 2018)

36. Sasaki, A. et al. Modeling of radiative properties of Sn plasmas for extremeultraviolet source. J. Appl. Phys. 107, 113303 (2010).

37. D'Arcy, R. et al. Transitions and the effects of configuration interaction in the spectra of Sn XV-Sn XVIII. Phys. Rev. A 79, 042509 (2009).

38. Schupp, R. et al. Efficient generation of extreme ultraviolet light from $\mathrm{Nd}$ : YAG-driven microdroplet-tin plasma. Phys. Rev. Appl 12, 014010 (2019).

39. Meijer, R. A., Stodolna, A. S., Eikema, K. S. E. \& Witte, S. High-energy Nd: YAG laser system with arbitrary sub-nanosecond pulse shaping capability. Opt. Lett. 42, 2758-2761 (2017).

40. Goh, S. et al. Fabrication and characterization of free-standing, high-linedensity transmission gratings for the vacuum UV to soft X-ray range. Opt. Express 23, 4421-4434 (2015).

41. Scott, H. A. In Modern Methods in Collisional-Radiative Modeling of Plasmas (ed. Ralchenko, Y.), 81-104 (Springer International Publishing, Cham, 2016)

42. Basko, M. M., Sasorov, P. V., Murakami, M., Novikov, V. G. \& Grushin, A. S. One-dimensional study of the radiation-dominated implosion of a cylindrical tungsten plasma column. Plasma Phys. Control. Fusion 54, 055003 (2012).

43. Bleiner, D. et al. (eds.) Short Wavelength Laboratory Sources (The Royal Society of Chemistry, 2015)

44. Legall, $\mathrm{H}$. et al. Compact $\mathrm{x}$-ray microscope for the water window based on a high brightness laser plasma source. Opt. Express 20, 18362-18369 (2012).

45. Ohashi, H. et al. Quasi-moseley's law for strong narrow bandwidth soft x-ray sources containing higher charge-state ions. Appl. Phys. Lett. 104, 234107 (2014).

46. Griem, H. R. Validity of local thermal equilibrium in plasma spectroscopy. Phys. Rev. 131, 1170-1176 (1963).
47. Bauche, J., Bauche-Arnoult, C. \& Peyrusse, O. Atomic Properties in Hot Plasmas (Springer International Publishing, 2015)

48. Nikiforov, A. F., Novikov, V. G. \& Uvarov, V. B. Quantum-Statistical Models of Hot Dense Matter: Methods for Computation Opacity and Equation of State, vol. 37 (Springer Science \& Business Media, 2006)

49. Basko, M. M., Maruhn, J. \& Tauschwitz, A. Development of a 2D radiationhydrodynamics code RALEF for laser plasma simulations, GSI Report 2010-1, PLASMAPHYSICS- 25 (GSI Helmholtzzentrum flur Schwerionenforschung $\mathrm{GmbH}, 2010)$.

50. Kurilovich, D. et al. Power-law scaling of plasma pressure on laser-ablated tin microdroplets. Phys. Plasmas 25, 012709 (2018).

\section{Acknowledgements}

We thank J. Abdallah, C.J. Fontes, H.A. Scott, Y.R. Frank and J.W.M. Frenken for useful discussions. This project has received funding from European Research Council (ERC) Starting Grant number 802648 and is part of the VIDI research programme with project number 15697, which is financed by the Netherlands Organization for Scientific Research (NWO). Part of this work has been carried out at the Advanced Research Center for Nanolithography (ARCNL), a public-private partnership of the University of Amsterdam (UvA), the Vrije Universiteit Amsterdam (VU), NWO and the semiconductor equipment manufacturer ASML. Part of this work was supported by the Physics \& Engineering Materials (PEM) program of the US Department of Energy through the Los Alamos National Laboratory. Los Alamos National Laboratory is operated by Triad National Security, LLC, for the National Nuclear Security Administration of U.S. Department of Energy (Contract No. 89233218NCA000001). The spectrometer has been supported by the FOM Valorisation Prize 2011 awarded to F. Bijkerk and NanoNextNL Valorization Grant awarded to M. Bayraktar in 2015.

\section{Author contributions}

A.N. and J.C. performed the atomic structure and opacity calculations. R.S., R.M., and F.T. performed the experiment for which M.B. provided the spectrometer. J.S. and M.M. B. performed radiation-hydrodynamics simulations. F.T. analyzed calculations, simulations, and experimental results. F.T., J.S., J.C., and O.V. drafted the paper assisted by S.W., W.U., and R.H. All authors reviewed the paper.

\section{Competing interests}

The authors declare no competing interests.

\section{Additional information}

Correspondence and requests for materials should be addressed to O.O.V. or J.C.

Peer review information Nature Communications thanks the anonymous reviewers for their contribution to the peer review of this work.

Reprints and permission information is available at http://www.nature.com/reprints

Publisher's note Springer Nature remains neutral with regard to jurisdictional claims in published maps and institutional affiliations.

Open Access This article is licensed under a Creative Commons Attribution 4.0 International License, which permits use, sharing, adaptation, distribution and reproduction in any medium or format, as long as you give appropriate credit to the original author(s) and the source, provide a link to the Creative Commons license, and indicate if changes were made. The images or other third party material in this article are included in the article's Creative Commons license, unless indicated otherwise in a credit line to the material. If material is not included in the article's Creative Commons license and your intended use is not permitted by statutory regulation or exceeds the permitted use, you will need to obtain permission directly from the copyright holder. To view a copy of this license, visit http://creativecommons.org/ licenses/by/4.0/

(C) The Author(s) 2020 